\title{
ESTRATEGIAS DE AFRONTAMIENTO AL ESTRÉS EN RELACIÓN CON LA INTELIGENCIA EMOCIONAL EN NIÑOS DE 7 A 12 AÑOS DE EDAD VÍCTIMAS DE DESASTRE
}

\author{
COPING STRATEGIES TO STRESS ABOUT EMOTIONAL INTELLIGENCE IN \\ CHILDREN 7 TO 12 YEARS OF DISASTER VICTIMS
}

\author{
LUIS ECHAVARRIA R. ${ }^{1}$ \\ Universidad Nacional Mayor de San Marcos, Lima, Perú \\ (ReCibido el 19/1/2012; ACEPTAdo el 15/6/2012)
}

\begin{abstract}
RESUMEN
Este trabajo de investigación estudia la relación entre las estrategias de afrontamiento al estrés y la inteligencia emocional en una muestra de 227 niños víctimas de desastre, de ambos sexos, cuyas edades fluctúan entre 7 y 12 años de edad. Para establecer la relación entre dichas variables se utilizó el diseño correlacional; en tanto para la recolección de datos, se aplicó la Escala de Estrategias de Afrontamiento al Estrés en Niños, la que fue creada por el investigador, cuya validez se estableció mediante el criterio de jueces y la confiabilidad a través del análisis interno donde se obtuvo un alfa de Cronbach de .787 para la Estrategia de Afrontamiento Centrado en el Problema y de .696 para la Estrategia de Afrontamiento Centrado en la Emoción; así mismo, se empleó el Inventario de Inteligencia Emocional BarOn ICE:NA, que fue adaptado y estandarizado a una muestra limeña por Ugarriza y Pajarez (2003). Los resultados del análisis de correlación Rho de Spearman indicaron que existe una relación estadísticamente significativa entre las estrategias de afrontamiento al estrés centradas en el problema y el cociente emocional total, siendo la misma de .36 ( $<<$ .01 ); igualmente, se encontró una relación significativa y moderada entre las estrategias de afrontamiento centradas en la emoción y el cociente emocional total, con una correlación de $.41(\mathrm{p}<.01)$.
\end{abstract}

Palabras clave: Estrategias de afrontamiento al estrés, cociente emocional, niños víctimas de desastre.

1 Psicólogo Clínico del Área de Diagnóstico y Tratamiento Neuropsicológico del Instituto Psicopedagógico EOS - Perú I lechavarria@eosperu.net / Imer212@hotmail.com 


\begin{abstract}
This research examines the relationship between stress coping strategies and emotional intelligence in a sample of 227 child victims of disaster, of both sexes, ages 7 to 12 years of age. To establish the relationship between these variables using correlational design, in both data collection was applied Coping Strategies Scale Stress in Children, the same that was created by the researcher, whose validity was established by the criterion of judges and reliability through internal analysis which yielded a Cronbach alpha of .787 for the Coping Strategy Focused on the Problem and .696 for the Coping Strategy Focused on Emotion, likewise, we used the Inventory ICE BarOn Emotional Intelligence: NA, it was adapted and standardized for a sample limeña Ugarriza and Pajarez (2003). The results of correlation analysis of Spearman Rho, indicated a statistically significant relationship between stress coping strategies focused on the problem and total emotional quotient, with the same .36 (p $<.01$ ), likewise, found a significant relationship between the moderate and coping strategies focused on emotion and emotional quotient total, with a correlation of $0.41(\mathrm{p}<.01)$.
\end{abstract}

Keywords: Coping with stress, emotional quotient, child victims of disaster.

\title{
INTRODUCCIÓN
}

Los desastres son eventos súbitos de gran destrucción que limitan el tiempo y sus efectos pueden ser duraderos y públicos, esto incluye los embates de la naturaleza, accidentes tecnológicos por errores humanos, actos violentos impredecibles, entre otros (APA, 1983, citado por Valero, 2002).

El daño de los desastres se puede observar fácilmente en función de las pérdidas materiales; sin embargo, esto puede generar una crisis sobre la estructura social, económica, política y cultural de las comunidades y, por ende, perjudicar el normal desarrollo individual, familiar o social de las personas y principalmente el de los niños (Williams, 2005).

El estar expuestos a una situación de desastre pone en peligro la vida de las personas, haciendo que ellos muestren diferentes reacciones, siendo estas normales ante una situación anormal (Valero, 2002). No obstante, tales reacciones pueden generar en el sujeto un estrés repentino con dificultades para poder aceptar su nueva realidad y las respuestas emocionales que da, carecen de control y son exteriorizadas de manera intensa y hasta cierto punto inadecuada.

Siendo los desastres una situación en particular, ya que afectan la tríada biopsicosocial de los seres humanos, merecen mucha atención los problemas emocionales; puesto que el impacto sobre la emocionalidad de las personas y en especial la de los niños, ya que su personalidad se encuentra en desarrollo, varía en el tiempo, acentuándose o sobrepasando su capacidad de control en los primeros momentos después del evento (De Taborda, 2006).

Uno de los más remotos constructos teóricos sobre el estrés como respuesta propuesto por Janis en 1954, considera tres segmentos para estudiar al estrés en situaciones adversas: El evento desastroso, la respuesta psicológica por parte de los individuos y los determinantes intrapsíquicos y situacionales a tales respuestas (Valero, 2002). 
Igualmente, se debe de indicar que ante un desastre o emergencia, se presenta en el individuo un estado temporal de trastorno y desorganización caracterizado principalmente por la incapacidad para abordar situaciones particulares utilizando métodos acostumbrados para la solución de problemas y por el potencial para obtener un resultado radicalmente positivo o negativo, al cual se le llama crisis.

Por ello, se debe precisar que los patrones de enfrentamiento durante una situación de crisis son diferentes de aquellos relacionados con el estrés, puesto que durante la crisis parece ser que el enfrentamiento es "más personal" usando menos núcleo familiar y los apoyos informales. Así mismo, la bibliografía precisa que durante el estado de crisis, el individuo tiende a ser menos defensivo y más abierto a las sugerencias, influencias y apoyos; siendo ello, no característico del estrés (Valero, 2002).

Considerando lo descrito y teniendo en cuenta el punto de vista de la salud mental, las emergencias y desastres implican una perturbación social que sobrepasa la capacidad de afrontamiento o manejo de la población afectada. Según Lazarus y Folkman (1984), el afrontamiento viene a ser aquellos esfuerzos cognitivos y conductuales constantemente cambiantes que se desarrollan para manejar las demandas específicas externas y/o internas que son evaluadas como excedentes de los recursos del individuo.

En cuanto al afronte de los niños, la UNICEF (1985) proporciona una serie de estrategias que orientan al trabajo con niños víctimas de situaciones de accidentes o desastres. Dicho manual indica que los niños expresan y reproducen las experiencias dolorosas a través del juego y otras formas de comunicación, los recursos provienen de las redes de soporte social que amortiguan los efectos de la situación traumática (Valero, 2002).

Se conoce mucho acerca de la capacidad de adaptación de los niños, ya que poseen una personalidad en formación y una potencialidad innata de aprendizaje, están en continuo desarrollo y deben de enfrentar a diario nuevos retos, exigencias y necesidades que los llevan hacia el crecimiento y las adquisiciones cognitivas y afectivas. Esto supone una gran ventaja sobre los adultos en cuanto a su posibilidad de adaptación y afrontamiento a situaciones nuevas y esta capacidad de los niños debe aprovecharse en situaciones de crisis, pues ellos entienden que estas situaciones son difíciles, ven el peligro y reaccionan, pero así mismo, aprenden de lo que viven y de lo que ven (Casas, 2006).

Respecto a lo señalado anteriormente, se puede inferir que la capacidad de adaptación depende mucho de los recursos que el medio le brinda al niño para que logre un adecuado afronte.

Esto implicaría que las estrategias de afrontamiento al estrés podrían verse influidas por el nivel del cociente emocional, haciendo que estas se vean incrementadas o decrementadas; teniendo además mayor incidencia sobre los niños víctimas de desastre, debido a las características de personalidad que se presentan en dicha etapa y a consecuencia de tal evento. 
Finalmente, las estrategias de afrontamiento al estrés y la inteligencia emocional, son fundamentales puesto que orientan y dirigen la vida de los niños, particularmente en situaciones de emergencia y/o desastre; por ello, el problema de investigación se plantea por medio de las siguientes preguntas: ¿Cuál es la relación entre las estrategias de afrontamiento al estrés centradas en el problema y la inteligencia emocional en niños de 7 a 12 años de edad víctimas de desastre? y ¿Cuál es la relación entre las estrategias de afrontamiento al estrés centradas en la emoción y la inteligencia emocional en niños de 7 a 12 años de edad víctimas de desastre?

\section{Definición de términos}

\section{Desastres}

"Evento relativamente súbito de gran destrucción, limitado en el tiempo, no obstante, sus efectos pueden ser duraderos y públicos (afectan a más de una familia), esto incluye las fuerzas de la naturaleza, huracanes, terremotos, accidentes tecnológicos por error humano, accidentes de aviación, contaminaciones tóxicas, impredecibles actos de violencia entre otros" (American Psychological Association, División 12, Sección 1, 1989, citado por Valero, 2002, p. 19).

Estrés

Baum (1990, citado por Taylor, 2007) señala que el estrés es aquella experiencia emocionalmente negativa acompañada de cambios predecibles a nivel bioquímico, fisiológico, cognitivo y conductual y que están dirigidos ya sea para alterar el evento estresante o para adecuarse a sus efectos.

Estrategias de afrontamiento al estrés

Son esfuerzos cognitivos que están en constante cambio y que se desarrollan para manejar las demandas específicas (estresores), ya sean externas o internas, que son evaluadas como que exceden o desbordan los recursos que se poseen (Lazarus y Folkman, 1984); pueden ser centrados en el problema o centrados en la emoción.

Inteligencia emocional

De acuerdo al modelo desarrollado en 1977 por Bar-On, la inteligencia emocional es definida como un conjunto de habilidades personales, emocionales, sociales y de destreza que influyen en la habilidad para adaptarse y enfrentarse a las demandas y presiones del medio ambiente (Abanto, Higueras y Cueto, 2000, Ugarriza, 2003).

\section{MÉTODO}

Nivel y tipo de investigación

La investigación es un estudio descriptivo debido a que se trata de describir un fenómeno o situación mediante el estudio del mismo en una circunstancia témporoespacial determinada. Se trató de recoger información sobre el estado actual del fenómeno (Sánchez y Reyes, 2006).

El tipo de investigación es sustantivo descriptivo, pues estuvo orientada al conocimiento actualizado de las estrategias de afrontamiento al estrés y la inteligencia emocional en niños de 7 a 12 años de edad víctimas de desastre. 


\section{Diseño de investigación}

El diseño utilizado es correlacional porque se establecieron correlaciones entre las variables para determinar el grado en el que se relacionaron en un tiempo y en un grupo de estudiantes (Sánchez y Reyes, 2006).

En este estudio se sometieron a correlación las variables estrategias de afrontamiento al estrés e inteligencia emocional, en una muestra de niños víctimas de desastre de colegios nacionales del sur del país, para detectar la existencia de relaciones funcionales significativas que posibilitarán identificar en qué medida ambos grupos de variables se correlacionaban.

\section{Variables}

De acuerdo al tipo y al diseño de investigación, las variables correlacionadas fueron: Estrategias de afrontamiento al estrés e Inteligencia emocional.

Población y Muestra de Estudio

Población

Conformada por niños que estuvieron expuestos a una situación de desastre (terremoto o sismo durante el año 2007), de ambos sexos de las ciudades de Chincha, Pisco e Ica.

\section{Muestra}

El muestreo empleado fue de tipo no probabilístico (Sánchez y Reyes, 2006), dentro de su modalidad intencional, para lo cual se utilizó los siguientes criterios de selección de la muestra en pos de considerarla representativa mediante la inclusión de individuos típicos de la población (Sánchez y Reyes, 2006).

- $\quad$ Edad: entre 7 y 12 años

- Grados de escolaridad: que se encontraran cursando del $3^{\circ}$ al $6^{\circ}$ grado de educación primaria.

- $\quad$ Niños que fueron víctimas de desastre.

Se evaluó un total de 313 niños, de los cuales 86 evaluaciones fueron depuradas debido a que 4 excedían en edad y el resto (82) alcanzó un índice de inconsistencia mayor a lo establecido en el BarOn ICE:NA; quedando conformada la muestra por 227 niños, considerando 56 de la localidad de Ica, 103 de Pisco y 78 de Chincha.

Instrumentos y Técnicas de Recolección de Datos

La técnica que se utilizó para la colecta de datos fue diferente según las distintas variables.

- Estrategias de afrontamiento al estrés

Se elaboró un instrumento en base al modelo explicativo del estrés de Lazarus y Folkman (1986), quienes plantearon el afrontamiento como un proceso en lugar de rasgo; lo cual implica que las demandas -internas y externas- son evaluadas como excedentes o desbordantes de los recursos del individuo. 
En función de ello, se consideraron dos tipos de estrategias de afrontamiento al estrés, una centrada en el problema las que están dirigidas a la definición del problema, a la búsqueda de soluciones alternativas, a la consideración de tales alternativas en base a su costo y beneficio y a su elección y aplicación; y la otra centrada en la emoción en el que se considera a un grupo de procesos cognitivos encargados de disminuir el grado de trastorno emocional e incluyen estrategias como la evitación, la minimización, el distanciamiento, la atención selectiva, las comparaciones positivas y la extracción de valores positivos a los acontecimientos negativos.

\section{Ficha Técnica}

Nombre original : Escala de Estrategias de Afrontamiento al Estrés en Niños - EEAEN.

Autor : Luis Miguel Echavarría Ramírez

Año : 2008

Procedencia : Lima-Perú

Administración : Individual o Colectiva.

Aplicación : Niños y adolescentes de 7 a 18 años

Duración : 20 a 25 minutos

Significación : Se consideran dos tipos generales de estrategias de afrontamiento al estrés: orientadas al problema y orientadas a la emoción.

\section{Confiabilidad}

En el análisis de esta escala, los ítemes que evalúan las estrategias de afrontamiento al estrés centradas en el problemas, obtuvieron un Alfa de .787; mientras que los ítemes que exploran las estrategias de afrontamiento al estrés centradas en la emoción, lograron un Alfa de .673; ello indica que el instrumento es confiable.

\section{Validez}

La EEAEN fue sometida a criterio de jueces, donde 10 expertos dieron su sentencia a favor de lo que pretende medir dicho instrumento.

- Inventario Del Cociente Emocional Baron

Se aplicó el Inventario del Cociente Emocional de BarOn, adaptada a Lima por Ugarriza (2003). 


\section{Ficha Técnica}

Nombre original : EQi - YV BarOn Emotional Quotient Inventory

Autor $\quad$ : Reuven Bar - On
Procedencia $\quad$ : Toronto - Canadá
Adaptación Peruana: Nelly Ugarriza y Liz Pajares

Administración : Individual o Colectiva.

Aplicación : Niños y adolescentes de 7 a 18 años

Formas : Completa y abreviada

Duración : Sin límite de tiempo (forma completa: 20 a 25 minutos y abreviada de 10 a 15 minutos)

\section{Confiabilidad}

Ugarriza y Pajares (2003) estudiaron la confiabilidad en una muestra limeña, haciendo uso del método de la consistencia interna, donde hallaron que los coeficientes de confiabilidad fueron bastante satisfactorios a través de los diferentes grupos normativos en función al sexo, a la edad y gestión del centro escolar (estatal - particular), pese a que algunas escalas contienen poco número de ítemes. Así mismo, emplearon el estadístico media de las correlaciones inter-ítemes, encontrando que las medias de las correlaciones ofrecían un patrón similar a través de los diversos grupos normativos, brindando un soporte adicional para la consistencia interna de las escalas del inventario, mejorando con el incremento de la edad.

\section{Validez}

Para la muestra normativa peruana, en el Manual de la Adaptación y Estandarización a una muestra limeña se señala que la validación del test se ha centrado en dos asuntos principales: 1) la validez de constructo del inventario, y 2) la multidimensionalidad de las diversas escalas; sosteniéndose que este inventario tiene suficiente validez de constructo que garantiza su publicación y recomendación para uso clínico.

\section{RESULTADOS}

El análisis de los datos de las pruebas administradas a la muestra permitió apreciar que la edad de 10 años es aquella que divide al total por la mitad (tabla 1); del mismo modo, se aprecia que la edad más común es de 11 años, la que representa el $34.4 \%$ de los participantes (tabla 2). 
Tabla 1. Distribución de estadísticos descriptivos de la muestra según edad

\begin{tabular}{ccc}
\hline \multicolumn{3}{c}{ Estadísticos descriptivos } \\
Mediana & 10.00 \\
Moda & 11.00 \\
\hline n $=227$ & & \\
Tabla 2. Distribución de frecuencias y porcentajes de la edad de los participantes \\
\hline Edad & $\mathbf{f}$ & $\%$ \\
\hline 7 & 1 & 0.44 \\
8 & 26 & 11.45 \\
9 & 47 & 20.70 \\
10 & 52 & 22.91 \\
11 & 78 & 34.36 \\
12 & 23 & 10.13 \\
\hline Total & 227 & 100.0 \\
\hline
\end{tabular}

Por otro lado, en la Tabla 3, se aprecia que no hay varones que tengan 7 años; así mismo, se observa que existe la misma cantidad de niños y niñas que tienen 8 años (13). Igualmente, es preciso indicar que no existe mucha diferencia entre la cantidad total de varones y mujeres (111 y 116).

Tabla 3. Distribución de frecuencias de la edad según la variable sexo

\begin{tabular}{|c|c|c|c|c|c|c|c|}
\hline \multirow{2}{*}{ Sexo } & \multicolumn{6}{|c|}{ Edad } & \multirow{2}{*}{ Total } \\
\hline & 7 & 8 & 9 & 10 & 11 & 12 & \\
\hline Varón & 0 & 13 & 19 & 27 & 41 & 11 & 111 \\
\hline Mujer & 1 & 13 & 28 & 25 & 37 & 12 & 116 \\
\hline Total & 1 & 26 & 47 & 52 & 78 & 23 & 227 \\
\hline
\end{tabular}

En la Figura 1, se aprecia la distribución de los porcentajes según la edad de los niños; mientras que en la figura 2, se tiene la distribución de las frecuencias de la edad según la variable sexo. Si hacemos referencia a los porcentajes, se observa que sólo el $0.44 \%$ de los entrevistados poseen una edad de 7 años; en tanto que 41 varones tienen 11 años de edad. 


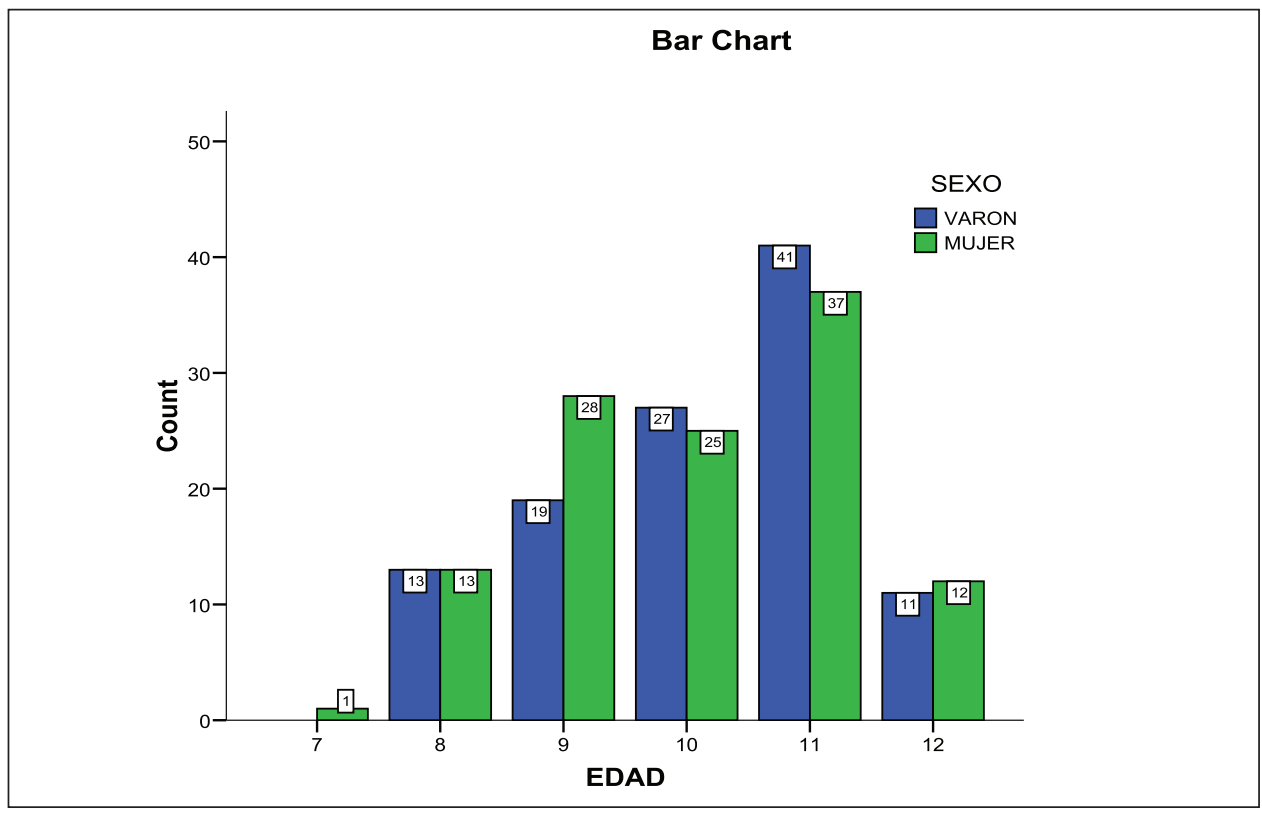

Figura1: Distribución de frecuencias de la edad según la variable sexo

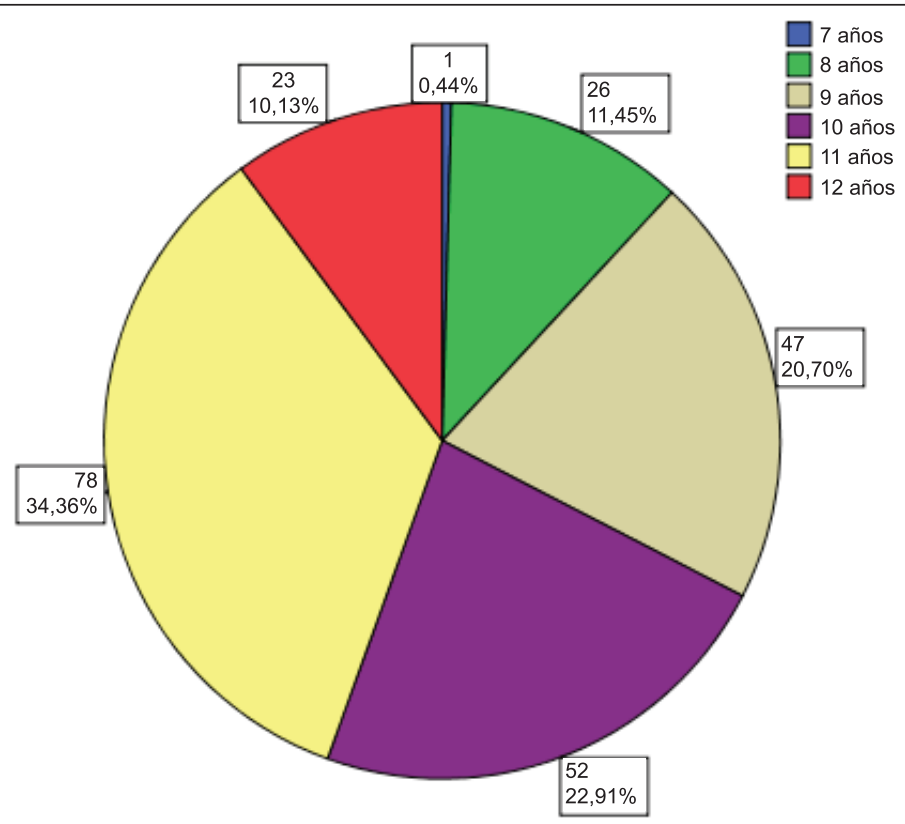

Figura 2: Distribución de frecuencias y porcentajes, según edad 
$\mathrm{Al}$ analizar la distribución de frecuencias y porcentajes del tipo de estrategia de afrontamiento al estrés que emplean los niños (ver tabla 4 y figura 3 ), se puede observar que las estrategias centradas en el problema alcanzan un porcentaje de $57.7 \%$, lo que en comparación a las estrategias de afrontamiento centradas en la emoción que logran un $37.0 \%$, indicaría que los niños hacen un mayor uso de las estrategias de afrontamiento centradas en el problema. Sin embargo, se pudo apreciar que solo un $5.3 \%$ de la muestra emplea los dos tipos de estrategias.

Tabla 4. Distribución de frecuencias y porcentajes, según el tipo de estrategia de afrontamiento utilizada

\begin{tabular}{ccc}
\hline Tipo de estrategia & $\mathrm{f}$ & $\%$ \\
\hline Centrada en el Problema & 131 & 57.7 \\
Centrada en la Emoción & 84 & 37.0 \\
Ambos Tipos & 12 & 5.3 \\
\hline Total & 227 & 100.0 \\
\hline
\end{tabular}

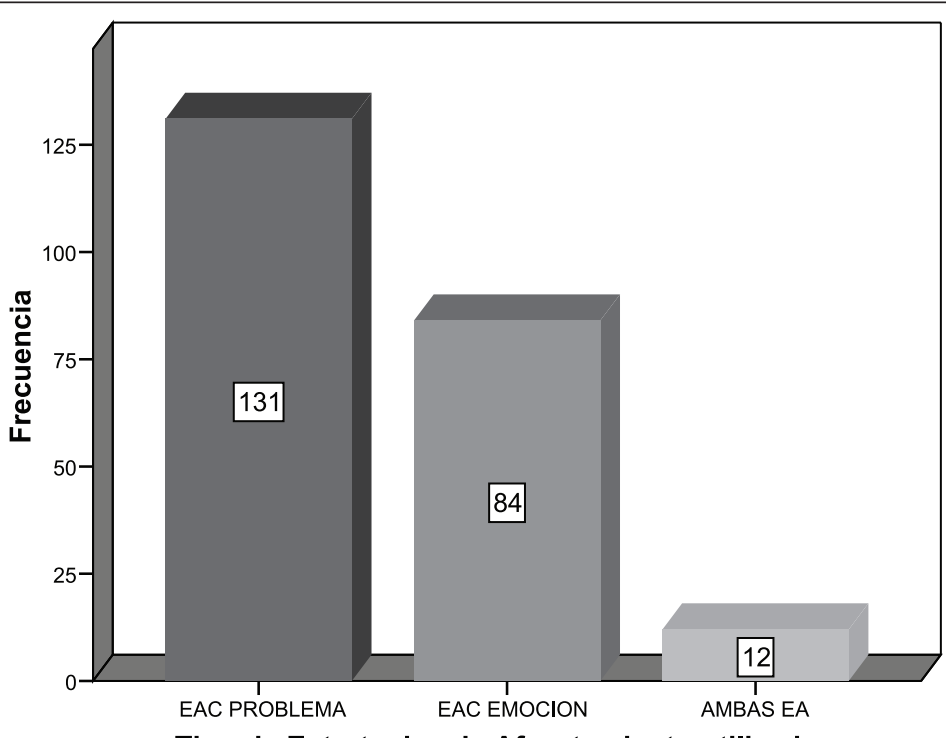

Tipo de Estrategias de Afrontamiento utilizadas

Figura 3: Distribución de frecuencias y porcentajes, según el tipo de estrategia utilizada

En la Figura 4 se observa la distribución de frecuencias según el tipo de uso de las estrategias de afrontamiento y la variable sexo, la cual se complementa con la tabla 5, en donde además se tiene la distribución de porcentajes de lo antes señalado. En ellos se puede apreciar que el $59.5 \%$ de los varones hace uso de estrategias centradas en el problema; mientras que el $56 \%$ de las mujeres emplea dicha estrategia. Si dichos porcentajes se trasladan en frecuencias, se observa que no existen diferencias entre la cantidad de varones (66) y el de las mujeres (65). 


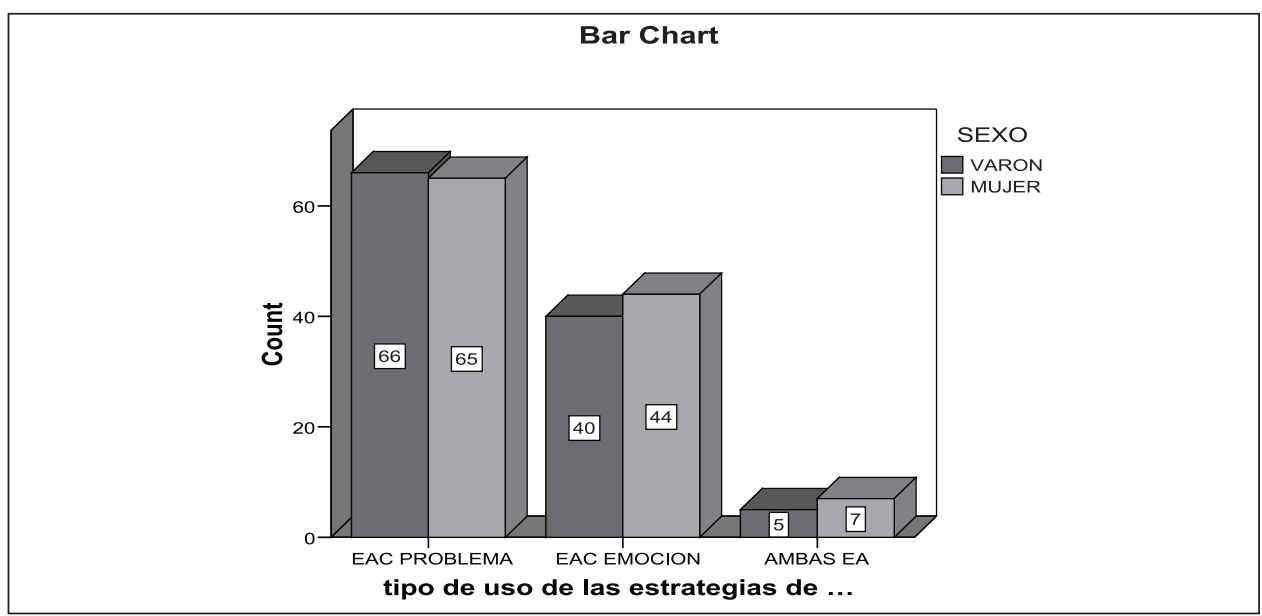

Figura 4: Distribución de frecuencias, según el tipo de uso de las estrategias utilizadas considerando y la variable sexo

Si se observa la Tabla 5, se puede apreciar que en relación con el uso de ambas estrategias, se tiene que 5 varones emplean los dos tipos de estrategias en comparación a las 7 mujeres que lo emplean; ello permite inferir que tampoco existen diferencias en las frecuencias.

Tabla 5. Distribución de frecuencias y porcentajes del tipo de estrategias de afrontamiento utilizadas según la variable sexo

\begin{tabular}{lccccccc}
\hline \multirow{2}{*}{ Estrategias de afrontamiento } & \multicolumn{2}{c}{ Varón } & \multicolumn{2}{c}{ Mujer } & \multicolumn{2}{c}{ Total } \\
& f & \% & f & $\%$ & f & $\%$ \\
\hline Centradas en el problema & 66 & 59.5 & 65 & 56.0 & 131 & 57.7 \\
Centradas en la emoción & 40 & 36.0 & 44 & 37.9 & 84 & 37.0 \\
Ambas estrategias de afrontamiento & 5 & 4.5 & 7 & 6.0 & 12 & 5.3 \\
\hline \multicolumn{1}{c}{ Total } & 111 & 100.0 & 116 & 100.0 & 227 & 100.0 \\
\hline
\end{tabular}

Respecto a las hipótesis generales, en la Tabla 6 podemos apreciar de manera universal la relación entre las estrategias de afrontamiento al estrés según el estilo, centrado en el problema o en la emoción, y el cociente emocional total.

Tabla 6. Relación entre las estrategias de afrontamiento al estrés según el estilo de afrontamiento (centrado en el problema o en la emoción) y el cociente emocional total, utilizando el coeficiente de correlación Rho de Spearman

\begin{tabular}{cccc}
\hline & $\begin{array}{c}\text { Cociente emocional } \\
\text { total }\end{array}$ & $\mathrm{n}$ & $\mathrm{p}$ \\
\hline $\begin{array}{c}\text { Estrategias de afrontamiento centradas en } \\
\text { el problema }\end{array}$ & $.36^{* *}$ & 131 & Significativa \\
$\begin{array}{c}\text { Estrategias de afrontamiento centradas en } \\
\text { la emoción }\end{array}$ & $.41^{* *}$ & 84 & Significativa \\
\hline
\end{tabular}


En cuanto a si existe relación entre las estrategias de afrontamiento al estrés centradas en el problema y el nivel de cociente emocional, se puede apreciar en la Tabla 7 que sí existe una correlación estadísticamente significativa entre dichas variables, siendo la misma de $.36(\mathrm{p}<.01)$, la misma que es una correlación positiva y moderada y que indica que el niño o niña que obtenga una puntuación mayor en el cociente emocional, tendrá una mayor preferencia de uso de las estrategias de afrontamiento al estrés centradas en el problema.

Tabla 7. Cálculo de la relación entre las estrategias de afrontamiento centradas en el problema y el cociente emocional total, utilizando el coeficiente de correlación Rho de Spearman

Cociente emocional total $\mathrm{n} \quad \mathrm{p}$

\begin{tabular}{llll}
\hline $\begin{array}{l}\text { Estrategias de afrontamien- } \\
\text { to centradas en el problema }\end{array}$ & $.36^{* *}$ & 131 & Significativa \\
\hline$* * \mathrm{p}<.01$ & &
\end{tabular}

Considerando la hipótesis que plantea la relación entre las estrategias de afrontamiento centradas en la emoción y el cociente emocional total, en la tabla 8 aprecia que dichas variables obtienen un coeficiente de $.41(\mathrm{p}<.01)$, el cual indica una correlación significativa positiva y moderada. Tal resultado permite inferir que los evaluados que alcancen un puntaje alto a nivel del cociente emocional tenderán a mostrar preferencia por emplear las estrategias de afrontamiento centradas en la emoción.

Tabla 8. Cálculo de la relación entre las estrategias de afrontamiento centradas en la emoción y el cociente emocional total, utilizando el coeficiente de correlación Rho de Spearman

Cociente emocional total $\mathrm{n} \quad \mathrm{p}$

\begin{tabular}{llll}
\hline $\begin{array}{l}\text { Estrategias de afrontamien- } \\
\text { to centradas en la emoción }\end{array}$ & $.41 * *$ & 84 & Significativa \\
$* * \mathrm{p}<.01$ & &
\end{tabular}

En la Tabla 9, se puede observar de manera genérica la correlación de las estrategias de afrontamiento al estrés centradas en el problema y los diversos componentes del inventario de inteligencia emocional.

Tabla 9. Cálculo de la relación entre las estrategias de afrontamiento centradas en el problema y los diversos componentes del inventario de inteligencia emocional, utilizando la prueba Rho de Spearman

\begin{tabular}{ccccc}
\hline & $\begin{array}{c}\text { Componente } \\
\text { intrapersonal }\end{array}$ & $\begin{array}{c}\text { Componente } \\
\text { interpersonal }\end{array}$ & $\begin{array}{c}\text { Componente } \\
\text { adaptabilidad }\end{array}$ & $\begin{array}{c}\text { Componente ma- } \\
\text { nejo del estrés }\end{array}$ \\
\hline $\begin{array}{c}\text { Estrategias de afronta- } \\
\text { miento centradas en el } \\
\text { problema }\end{array}$ & .16 & $.34^{* *}$ & $.37^{* *}$ & .06 \\
\hline $\mathrm{p}$ & No significativa & Significativa & Significativa & No significativa \\
\hline $\mathrm{n}=131$ & & & &
\end{tabular}




\section{DISCUSIÓN}

A lo largo de los análisis se ha podido comprobar la bondad de las escalas examinadas. Los resultados de los análisis psicométricos realizados sobre la Escala de Estrategias de Afrontamiento al Estrés muestran fiabilidad, puesto que los ítems que miden las estrategias centradas en el problema, alcanzaron un alfa de Cronbach de .79; mientras que, los ítems que valoran las estrategias centradas en la emoción, lograron un cociente de .67. De otro lado, el instrumento demostró validez a través del criterio de jueces.

Respecto al Inventario del Cociente Emocional de BarOn ICE:NA, se puede afirmar que alcanzó un cociente de correlación de .89; lo que implica que el test es confiable. Del mismo modo, la validez se estudió con el método de análisis factorial exploratorio, encontrándose que las escalas están conformando un solo factor y con una varianza explicada de $62.25 \%$.

La literatura señala que los estudios realizados desde la década de los ochenta distinguen entre los esfuerzos para alterar o modificar la situación estresante (afrontamiento centrado en el problema) y los esfuerzos para regular las consecuencias emocionales asociadas con la situación (afrontamiento centrado en la emoción) (Lazarus y Folkman, 1986). Así en la investigación se planteó la hipótesis de que existe relación entre las estrategias de afrontamiento al estrés centradas en el problema y el nivel de cociente emocional, siendo esta validada puesto que se aprecia la existencia de un índice de correlación positivo y significativo de .36; lo que permite sugerir que los niños de la muestra hacen uso de dichas estrategias de afrontamiento en relación al nivel de cociente emocional alcanzado por cada uno de ellos, al ser evaluados con el instrumento BarOn ICE:NA. Del mismo modo, se obtuvo una correlación positiva y moderada de .41 respecto a la relación entre las estrategias de afrontamiento al estrés centradas a la emoción y el cociente emocional total; lo que también permite colegir que el uso de la estrategia en mención se encuentra relacionada con el nivel de cociente emocional logrado por cada evaluado en la prueba mencionada.

Es decir, que lo antes descrito facilitaría interpretar que la situación estresante a la cual estuvieron sometidos los niños (desastre), conllevó a que realicen una evaluación de ella con la finalidad de buscar una solución alternativa o hacer uso de procesos cognitivos con el fin de evitar, minimizar, distanciarse del evento o dirigir su atención hacia algo más satisfactorio, generando un mayor uso de estrategias de afrontamiento al estrés centradas en el problema o en la emoción, en función del cociente emocional que alcancen en la prueba BarOn ICE:NA.

Teniendo en cuenta a BarOn (1977 citado por Ugarriza, 2003, Abanto, Higueras y Cueto, 2000), quien propuso que la inteligencia emocional es el conjunto de habilidades personales, emocionales, sociales y de destreza que influyen en la habilidad para adaptarse y enfrentarse a las demandas y presiones del medio; en la investigación se puede apreciar que no existe relación entre las estrategias de afrontamiento centradas en el problema y en la emoción con respecto a los componentes intrapersonal y manejo del estrés de la prueba de inteligencia emocional; 
mientras que sí se observa correlación con los componentes interpersonal y adaptabilidad. Ello permite sugerir que ante situaciones adversas, como es el caso de la ocurrencia de un desastre, los niños poseen estrategias para poder comprender, expresar y comunicar sus sentimientos y necesidades; además de tolerar tal circunstancia, enfrentando de manera pasiva y siendo poco tolerantes en el control de sus emociones. En relación con la muestra evaluada, se puede mencionar que esta carece de capacidades para poder adaptarse a su entorno, percatarse, comprender y apreciar los sentimientos de los demás; así como poder identificar y definir los problemas, no logrando evaluar la correspondencia entre lo que experimenta (subjetivo) y lo que en realidad existe (objetivo) (Ugarriza, 2003).

Además, a lo largo del proceso del estudio del estrés, se apreció que hay abundante literatura sobre el tema respecto a niños; no obstante y específicamente sobre el afrontamiento al estrés en situaciones de desastre, se conoce muy poco. Del mismo modo, también se ha visto que las intervenciones en situaciones de emergencia y desastre, indican que los niños presentan diferentes modos de reaccionar frente a éste y que su recuperación dependerá mucho de las estrategias de afrontamiento que use el niño y los medios que le ofrezca su entorno (Valero, 2002, Williams, 2005, De Taborda, 2006); por ello es importante señalar que los resultados de esta investigación deberán ser tomados como un acercamiento, pues se ha realizado tomando exclusivamente 227 participantes pertenecientes a un contexto específico (víctimas de desastre).

De otra parte, se planteó la relación entre las estrategias de afrontamiento centradas en el problema y el componente interpersonal del inventario BarOn ICE:NA, observándose de que el hecho de establecer adecuadas relaciones con las demás personas depende de las soluciones o alternativas, en base a su costo-beneficio y elección-aplicación, que tienen los evaluados (Lazarus y Folkman, 1986), de lo que se podría colegir que si un niño se siente seguro en un determinado espacio (albergue o refugio), no querrá salir de ahí e interactuará con quienes se encuentren a su alrededor, sean estos sus padres, cuidadores o personal sanitario.

Por otro lado, se puede inferir que las respuestas que dan los niños ante situaciones estresantes, tal es el caso de un desastre, por ejemplo: Llorar, gritar, sentir temor, dificultades para dormir, reaccionar exageradamente ante todo tipo de estímulo y difícilmente se tranquilizan, cambios en el comportamiento, rechazo al hablar o aislamiento (Casas, 2006); son respuestas normales e incluso esperadas ante una situación inesperada (Valero, 2002), no se relacionan con los procesos cognitivos que ellos poseen.

\section{CONCLUSIONES}

Al término de la investigación, se llegó a las siguientes conclusiones:

1. Existe una relación estadísticamente significativa entre las estrategias de afrontamiento centradas en el problema y el cociente emocional total.

2. Se encontró una relación estadísticamente significativa entre las estrategias de afrontamiento centradas en la emoción y el cociente emocional total. 
3. No existe relación estadística significativa entre las estrategias de afrontamiento al estrés centradas en el problema y el componente intrapersonal del inventario de inteligencia emocional.

4. Existe una relación estadística significativa, positiva y moderada entre las estrategias de afrontamiento al estrés centradas en el problema y el componente interpersonal de la prueba inteligencia emocional.

5. No se encontró correlación estadística significativa entre las estrategias de afrontamiento al estrés centradas en el problema y el componente manejo del estrés del inventario BarOn ICE:NA.

6. Existe una relación estadísticamente significativa entre las estrategias de afrontamiento al estrés centradas en el problema y el componente adaptabilidad de la prueba inteligencia emocional.

7. No existe relación estadística significativa entre las estrategias de afrontamiento al estrés centradas en la emoción y el componente intrapersonal del inventario de inteligencia emocional.

8. Se encontró una relación estadística significativa, positiva y moderada entre las estrategias de afrontamiento al estrés centradas en la emoción y el componente interpersonal de la prueba inteligencia emocional.

9. No se encontró correlación estadística significativa entre las estrategias de afrontamiento al estrés centradas en la emoción y el componente manejo del estrés del inventario BarOn ICE:NA.

10. Existe una relación estadísticamente significativa entre las estrategias de afrontamiento al estrés centradas en la emoción y el componente adaptabilidad de la prueba inteligencia emocional.

11. La Escala de Estrategias de Afrontamiento al Estrés en Niños, es válida y confiable.

12. La muestra tiene como preferencia de uso a las estrategias de afrontamiento centradas en el problema.

13. No se encontró diferencias en las frecuencias de uso de las estrategias de afrontamiento según la variable sexo.

\section{REFERENCIAS BIBLIOGRÁFICAS}

Abanto, Z., Higueras, L. y Cueto, J. (2000). Inventario de Cociente Emocional de BarOn - Manual Técnico. Lima: Editorial Grafimag.

Alarcón, R. (2008). Métodos y diseños de investigación del comportamiento. Lima: Universidad Ricardo Palma - Editorial Universitaria.

Ayvar, G. (1989). Condiciones de trabajo y nivel de estress laboral de las enfermeras del Servicio de Emergencias del Instituto Peruano de Seguridad Social Guillermo Almenara Irigoyen. Tesis para optar el título profesional de Licenciada en Enfermería. 
Obtenido el 15 de abril del 2009 en: http://www.cybertesis.edu.pe/sisbib/1989/ ayvar_pg/pdf/ayvar_pg.pdf

Bellock, A., Sandín, B. y Ramos, F. (1985). Manual de psicopatología Vol. 2. Madrid: Editorial Mc Graw Hill.

Casas, G. (2006). Atención psicosocial a la infancia y la adolescencia. Guía práctica de salud mental en situaciones de desastres. OPS/OMS. 97-125.

Delgado, A., Escurra, L. y Torres, W. (2006). La medición en Psicología y Educación: Teoría y Aplicaciones. Lima: Editorial Hozlo S.R.L.

De la Cruz, L. (2003). Afrontamiento al estrés en personas hipertensas del Centro del Adulto Mayor de Lima Cercado. Tesis para optar el Grado Académico de Magíster en Psicología con mención en Psicología Clínica y de la Salud. Obtenido el 20 de abril del 2009 en: http://www.cybertesis.edu.pe/sisbib/2003/delacruz_vl/pdf/delacruz_vlTH.back.1.pdf

De Taborda, M. (2006). Principales problemas psicosociales y principales pautas para su atención. Guía práctica de salud mental en situaciones de desastres. OPS/OMS. 43 - 71.

Escurra, L. (1989). Cuantificación de la validez de contenido por criterio de jueces. Revista de Psicología PUCP, 6(1-2). 103 - 111

Extremera, N. y Fernández, P. (2002). La inteligencia emocional: Métodos de evaluación en el aula. Universidad de Málaga. Obtenido el 24 de abril del 2009 en: http://www. rieoei.org/deloslectores/465Extremera.pdf

Fernández-Berrocal, P., Salovey, P., Vera, A., Ramos, N. y Extremera, N. (2001). Cultura, inteligencia emocional percibida y ajuste emocional: un estudio preliminar. Revista Electrónica de Motivación y Emoción 4(8-9)

Gardner, H. (1999). Las inteligencias múltiples - Estructura de la mente. Bogotá: Fondo de Cultura Económica.

Goleman, D. (1995). La inteligencia emocional. Buenos Aires: Javier Vergara Editor.

Gonzáles, J. (1994). Estrés, homeóstasis y enfermedad - Psicología Médica. Zaragoza: Ediciones A. Seva.

Hernández, R., Fernández, C. y Baptista, P. (2006). Metodología de la Investigación. México: Mc Graw Hill.

Lazarus, R. y Folkman, S. (1986). Estrés y procesos cognitivos. Barcelona: Martínez Roca.

Marín, H. (2005). Psicología de la emergencia: comportamiento humano antes, durante y después de emergencias. Trabajo presentado en el II Concgreso Nacional de Salud Mental y Asistencia Primaria en Catástrofes (Hopsital Central de la Defensa "Gómez Ulla" Madrid, 4 y 5 de Marzo de 2005), y en XIV Jornadas Municipales sobre Catástrofes Samur - Protección Civil (Madrid, del 24 al 26 de junio de 2005). Obtenido el 20 de enero de 2010 de:http://www.sld.cu/galerias/pdf/sitios/desastres/ introduccion_psicologia_emergencia_hmarin.pdf 
Maúrtua-Toro, C. (2007). La inteligencia emocional en niños con talento intelectual. Archivos Psicológicos - Revista de los Estudiantes de la Facultad de Psicología URP, 4(1), 59-71.

Mercado, G. (2006). Nivel de estrés laboral en enfermeras en el Hospital Nacional Dos de Mayo. Tesis para optar el título profesional de Licenciada en Enfermería. Obtenido el 10 de abril del 2009 en: http://www.cybertesis.edu.pe/sisbib/2006/mercado_tg/ pdf/mercado_tg-TH.4.pdf

Merino, C. (1999). Visión introductoria al estrés infantil. Obtenido el 18 de abril del 2009 en: http://www.psicopedagogia.com/articulos/?articulo $=177$

Merino, C., Manrique, G., Angulo, M. e Isla, N (2007). Indicador de Estrategias de Afrontamiento al Estrés: Análisis normativo y de su estructura factorial. Ansiedad y Estrés, 13(1), 25-40.

Nervi, V. (2007). Relación entre la inteligencia Emocional y el Rendimiento Académico en adolescentes de 15 y 16 años. Archivos Psicológicos - Revista de los Estudiantes de la Facultad de Psicología URP, 4(1), 73-80.

Paz, M., Álvarez, T. y Sánchez, A. (2003). Análisis de la relación entre inteligencia emocional, estabilidad emocional y bienestar psicológico. Universitas Psychologica, 2(001), 27-32.

Rivera, G (S/F). ¿Que hacer ante una emergencia...? - Guía practica: "primeros auxilios psicológicos”. Obtenido el 10 de enero del 2011 en: http://www.redem.org/ boletin/files/REDEM\%20- \%20Primeros \%20Auxilios \%20Psicolgicos \%202008\%20 (Tamao\%20minimo).pdf

Sánchez, H. y Reyes, C. (2006). Metodología y diseños en la investigación científica. Lima: Editorial Visión Universitaria.

Taylor, S. (2007). Psicología de la Salud - $6^{\circ}$ edición. México: Mc Graw Hill.

Ugarriza, N. (2003). Adaptación y estandarización del Inventario de Inteligencia Emocional de BarOn ICE:NA, en niños y adolescentes. Lima: Ediciones Libro Amigo.

Valero, S. (2002). Psicología en emergencias y desastres. Lima: Editorial San Marcos.

Williams, J. (2005). El desarrollo de la primera infancia en situaciones de emergencia. Espacio para la infancia, 23, 16 - 21. 
